\title{
SATURN FORMS BY CORE ACCRETION IN 3.4 Myr
}

\author{
Sarah E. Dodson-Robinson,,${ }^{1,2}$ Peter Bodenheimer,${ }^{3}$ Gregory Laughlin, ${ }^{3}$ Karen Willacy, ${ }^{4}$ \\ Neal J. Turner, ${ }^{4}$ and C. A. Beichman ${ }^{2}$ \\ Received 2008 July 15; accepted 2008 October 9; published 2008 November 3
}

\begin{abstract}
We present two new in situ core accretion simulations of Saturn with planet formation timescales of $3.37 \mathrm{Myr}$ (model S0) and $3.48 \mathrm{Myr}$ (model S1), consistent with observed protostellar disk lifetimes. In model S0, we assume rapid grain settling reduces opacity due to grains from full interstellar values. In model S1, we do not invoke grain settling, instead assigning full interstellar opacities to grains in the envelope. Surprisingly, the two models produce nearly identical formation timescales and core/atmosphere mass ratios. We therefore observe a new manifestation of core accretion theory: at large heliocentric distances, the solid core growth rate (limited by Keplerian orbital velocity) controls the planet formation timescale. We argue that this paradigm should apply to Uranus and Neptune as well.
\end{abstract}

Subject headings: planets and satellites: formation — planets and satellites: individual (Saturn)

\section{INTRODUCTION}

The discrepancy between the observed lifetimes of protostellar disks (2-3 Myr; Haisch et al. 2001) and the length of time required for planet formation by core accretion (>8 Myr; Pollack et al. 1996) has long presented a problem for planet formation theory. However, in 2005, two new models of planet formation showed that the core accretion-gas capture process could form gas giants within 2.5 Myr. Hubickyj et al. (2005) modeled Jupiter's formation in situ at 5.2 AU and found that Jupiter could grow from a $0.1 M_{\oplus}$ core to its present mass in 2.2 Myr. The core accretion models of Alibert et al. (2005) allowed both Jupiter and Saturn to form concurrently within $2.5 \mathrm{Myr}$ in disk of mass $0.035 M_{\odot}<M_{\text {disk }}<0.05 M_{\odot}$.

Each model employed a different approach in order to speed up giant planet formation. Alibert et al. added type I migration to the core accretion model: the inward motion of the protoplanets allows them to receive a fresh supply of planetesimals and gas as they move into undepleted regions of the solar nebula. The accretion rate is then no longer limited by the rate at which the protoplanet's Hill sphere expands, so the giant planet formation timescale decreases by up to an order of magnitude. These models require proto-Jupiter to have an initial semimajor axis of $a \geq 9.2 \mathrm{AU}$ and proto-Saturn to begin forming at $11.9 \mathrm{AU}$, and migrate to their current positions on a $\sim 2$ Myr timescale.

One notable feature of the Alibert et al. (2005) core accretion model is that it successfully predicts the heavy metal content of Jupiter and Saturn's atmospheres according the clathrate hydrate trapping theory of Lunine \& Stevenson (1985). However, the type I migration rate is a free parameter: Alibert et al. note that the analytical work of Tanaka et al. (2002) rates predicts migration rates far too large to be consistent with the observed frequency of extrasolar planets. The authors get around this problem by scaling the Tanaka et al. (2002) by an

\footnotetext{
${ }^{1}$ Formerly Sarah E. Robinson.

${ }^{2}$ NASA Exoplanet Science Institute, California Institute of Technology, 770 South Wilson Avenue, Pasadena, CA 91125; sdr@ipac.caltech.edu, chas@ipac.caltech.edu.

${ }^{3}$ University of California Observatories/Lick Observatory, Department of Astronomy and Astrophysics, University of California at Santa Cruz, Santa Cruz,CA 95064; peter@ucolick.org, laughlin@ucolick.org.

${ }^{4}$ Jet Propulsion Laboratory, California Institute of Technology, Pasadena, CA 91109; karen.willacy@jpl.nasa.gov, neal.turner@jpl.nasa.gov.
}

arbitrary factor $f_{1}$, where $0 \leq f_{1} \leq 0.03$. Finally, there is one more basic assumption underlying the model, which is that the gas/solid ratio beyond the ice line is constant at $G / S=70$.

The in situ planet formation models of Hubickyj et al. (2005) decrease Jupiter's formation time by requiring that grains quickly settle to the bottom of the protoplanetary envelope, where they are destroyed by sublimation (Podolak 2003). Assuming grain settling lowers envelope opacity due to grains to $\sim 2 \%$ of the interstellar value, the gas can contract efficiently and make way for new material entering the protoplanet's Hill sphere. The most important free parameter in the Hubickyj et al. models is the solid surface density of planetesimals in the planet's feeding zone: since the protoplanet does not move through the disk, it requires a feeding zone with $\Sigma_{\text {solid }} \gtrsim 10 \mathrm{~g}$ $\mathrm{cm}^{-2}$ in order to attain the $\sim 15 M_{\oplus}$ core necessary for accreting a massive gaseous envelope (Papaloizou \& Nelson 2005).

The availability of new calculations of solid surface density as a function of heliocentric distance in the solar nebula (Dodson-Robinson et al. 2008, hereafter Paper I) raises the possibility of extending in situ core accretion simulations to include Saturn. Since the Nice model of Tsiganis et al. (2005) predicts that proto-Saturn migrated $1 \mathrm{AU}$ outward at most, we consider the in situ approximation reasonable when applied to Saturn. By providing theoretically and observationally motivated values for solid surface density $\Sigma_{\text {solid }}$, the Paper I results move core accretion simulations away from parameter studies and toward determinism.

Since the solar nebula had to be capable of forming both Jupiter and Saturn concurrently (and of course Uranus and Neptune, the formation of which we will examine in future work), we first assess the ability of the Paper I solar nebula model to produce Jupiter. In Paper I, the value for $\Sigma_{\text {solid }}$ after $10^{5} \mathrm{yr}$ of solar nebula evolution is $13.2 \mathrm{~g} \mathrm{~cm}^{-2}$. Adopting the relationship between solid surface density and Jupiter formation time at 5 AU calculated by Robinson et al. (2006),

$$
\frac{t_{\text {form }}}{1 \mathrm{Myr}}=\left(\frac{\Sigma_{\text {solid }}}{25.0 \mathrm{~g} \mathrm{~cm}^{-2}}\right)^{-1.44}
$$

we find that the Paper I results allow Jupiter to form in 2.5 Myr. Built in to the Robinson et al. (2006) scaling relation is the assumption that efficient grain settling leads to protoplanetary envelope opacities of $\sim 2 \%$ those of interstellar grains. 

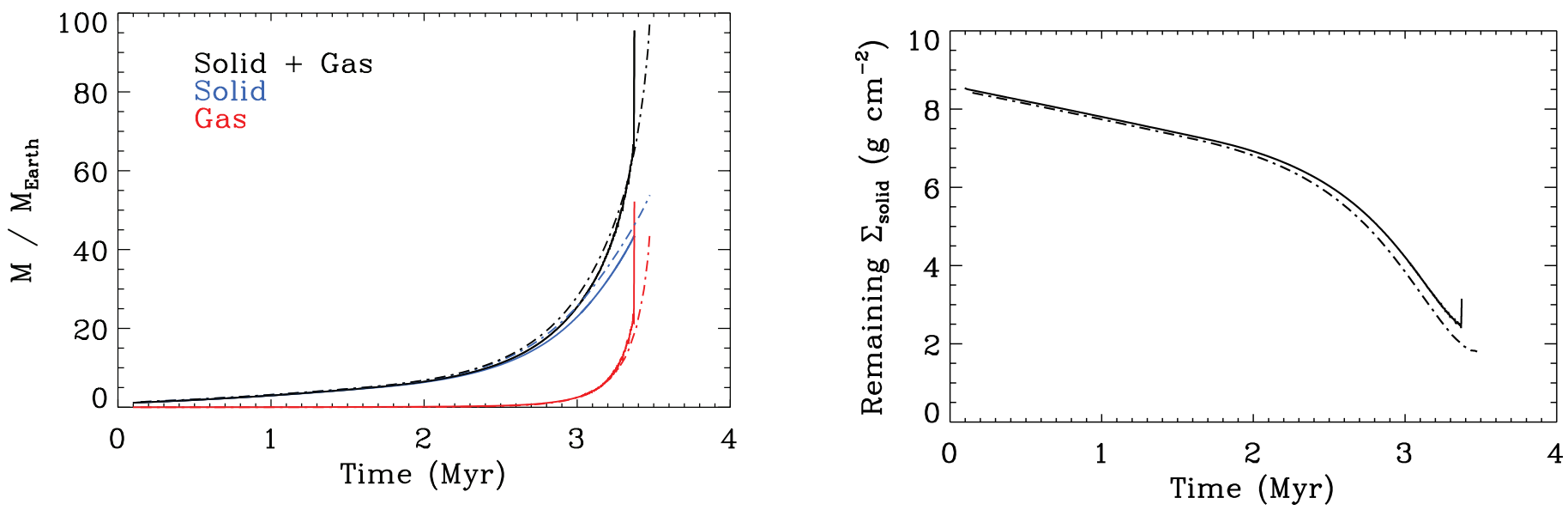

FIG. 1.-Left: Mass of Saturn as a function of time. Solid lines represent model S0 (reduced grain opacity) and dashed lines represent model S1 (full grain opacity). The black curves show the total planet mass, the blue curves show the solid mass only (presumed to be concentrated in the core), and the red curves show the gas mass. Saturn reaches its current mass, $95 M_{\oplus}$, in 3.4 Myr. Right: Remaining solid surface density, not yet accreted by Saturn, as a function of time. Solid line shows model S0 and dashed line shows model S1. The sharp upturn at $3.3 \mathrm{Myr}$ in S0 is due to the rapid expansion of Saturn's feeding zone when hydrodynamic gas accretion begins.

In this Letter, we will relax this assumption and also investigate the limiting case of $100 \%$ interstellar grain opacity with respect to Saturn.

If the fiducial disk from Paper I can form Saturn within 2$3 \mathrm{Myr}$, we will have successful core accretion models of the two gas giants forming near their present positions in a gravitationally stable disk (see Paper I for a discussion of the solar nebula dynamics).

In $\S 2$, we describe our theoretical treatment of the core accretion process. In $\S 3$, we discuss the results of our simulations, with special emphasis on formation timescale, effect of atmospheric opacity, and core/atmosphere mass ratio. We present our conclusions in $\S 4$.

\section{THEORETICAL TREATMENT OF CORE ACCRETION}

We use the theoretical model of planet formation described by Laughlin et al. (2004) to model the core accretion and gas capture of proto-Saturn. Initially, a protoplanetary core of mass $M_{\oplus}$ is embedded at Saturn's heliocentric distance, 9.5 AU, in a viscously evolving disk of age $1.5 \times 10^{5} \mathrm{yr}$, surrounding a T Tauri star of mass $1 M_{\odot}$. We assume that by $1.5 \times 10^{5} \mathrm{yr}$, the available dust has formed $100 \mathrm{~km}$ planetesimals that are invulnerable to gas drag (Weidenschilling 1977): planetesimal orbits are modified only by interactions with proto-Saturn. Gas temperature and density are regulated by viscous evolution of the solar nebula. We use the time-evolving temperature and density at $9.5 \mathrm{AU}$, beginning at $t=1.5 \times 10^{5} \mathrm{yr}$, as calculated in Paper I.

The contraction and buildup of protoplanetary cores and their gaseous envelopes embedded in our model evolving disk are computed with a Henyey-type code (Henyey et al. 1964). Following the argument of Podolak (2003) that grain settling in the protoplanetary envelope would reduce envelope opacity where grains exist, we adopt grain opacities of $2 \%$ of the interstellar values used in Pollack et al. (1996) in our fiducial model, which we will call S0. However, in order to assess the effect of envelope opacity on Saturn's formation timescale, we present a second core-accretion simulation, S1, using full interstellar grain opacity.
We use a core accretion rate of the form

$$
\frac{d M_{\text {core }}}{d t}=C_{1} \pi \Sigma_{\text {solid }} R_{c} R_{h} \Omega
$$

(Papaloizou \& Terquem 1999), where $\Sigma_{\text {solid }}$ is the surface density of solid material in the disk, $\Omega$ is the orbital frequency at 9.5 AU, $R_{c}$ is the effective capture radius of the protoplanet for solid particles, $R_{h}=a\left[M_{\text {planet }} /\left(3 M_{*}\right)\right]^{1 / 3}$ is the tidal radius of the protoplanet (where $a$ is the semimajor axis of the protoplanet's orbit), and $C_{1}$ is a constant near unity.

The outer boundary conditions for the protoplanet include the decrease with time in the background nebular density and temperature. During the late phase of planet growth, when planetesimals may be ablated by the massive envelope before reaching the core, we consider a planetesimal captured if it deposits $50 \%$ or more of its mass in the envelope. At this stage, we invoke the sinking approximation and assume the ablated planetesimal debris sinks rapidly to the planet core without leaving remnants in the envelope.

\section{RESULTS}

In both simulations ( $\mathrm{S} 0$ and $\mathrm{S} 1$ ), we start the core accretion model with midplane temperature, gas density and solid surface density from the solar nebula model of Paper I. This model has two key features favoring planet formation that are missing from passive disk models: (1) viscous stresses drive the initial $\Sigma \propto R^{-3 / 2}$ surface density profile toward uniformity, so that Saturn's feeding zone gains mass during the first $5 \times 10^{4} \mathrm{yr}$ of disk evolution, and (2) the presence of hydrated ammonia ice at the snow line increases the solid surface density by $7 \%$ over the standard water-ice-rock-refractory $\mathrm{CHON}$ mixture. We use a starting solid surface density of $8.6 \mathrm{~g} \mathrm{~cm}^{-2}$ (Paper I) which decreases with time as proto-Saturn captures planetesimals.

Figure 1 (left) shows the growth of Saturn from a core of 1 $M_{\oplus}$ to its present-day mass of $95 M_{\oplus}$. Solid lines correspond to the S0 model (in which grains quickly settle and sublimate, reducing their contribution to envelope opacity by an assumed factor of 50), and dashed lines show the S1 model (in which grains stay in the envelope and opacity due to grains takes on 
the full interstellar value). An important property of our model, and one of two key results of this Letter, is Saturn's formation time: the planet attains its current mass in only 3.37 Myr for model S0 and 3.47 Myr for model S1. These are the first in situ core accretion models of Saturn with formation times within $1 \sigma$ of observed protostellar disk lifetimes.

The second key result is that Saturn's formation time is nearly independent of the assumed grain opacity in the envelope. This surprising result occurs because Saturn's core growth rate is limited by the Keplerian speed in the feeding zone: a slowmoving core takes a long time to encounter and capture planetesimals (see eq. [2]), and may never reach isolation mass. Whereas during Jupiter's formation early core buildup takes only $0.5 \mathrm{Myr}$ and the gas contraction phase dominates planet growth (Hubickyj et al. 2005), in Saturn's case, core growth lasts until the planet reaches its final mass. Only in the last $6 \times 10^{5} \mathrm{yr}$ of growth does Saturn possess a gas mass of $>1 M_{\oplus}$.

Given that the planet has a negligible envelope mass throughout most of its formation, it stands to reason that envelope opacity would not exert much influence on Saturn's formation timescale. The idea that solid growth rate controls Saturn's formation is consistent with the planet's high core/atmosphere mass ratio: $9 \%-23 \%$, as opposed to $<3 \%$ for Jupiter (Saumon \& Guillot 2004).

\subsection{Core/Atmosphere Mass Ratio}

If all accreted planetesimals and their ablated debris end up in the solid core, as is assumed in our model, Saturn's total core mass reaches $44 M_{\oplus}$ in model S0 and $54 M_{\oplus}$ in model $\mathrm{S} 1$. Based on gravitational moment measurements and internal structure modeling, Saumon \& Guillot (2004) place Saturn's core at 9-22 $M_{\oplus}$ and total heavy element content at 13-28 $M_{\oplus}$. Our model Saturn has a heavy element mass that is too high by at least a factor of 1.6 (model S0). We note, however, that the carbon enrichment in Saturn's atmosphere has recently been revised upward to $(\mathrm{C} / \mathrm{H}) /(\mathrm{C} / \mathrm{H})_{\odot}=7$ (Flasar et al. 2005), which may allow for a larger heavy element inventory than previously thought.

One method of reducing the core mass, although not the total heavy element mass, is to account for planetesimal disruption not only by ablation of debris ( $\S 2$ ), but by sublimation of volatiles. Our planetesimals are $50 \% \mathrm{H}_{2} \mathrm{O}$ by mass, $6 \% \mathrm{NH}_{3}$, and $1 \%$ other ices, such as $\mathrm{HCN}$ and $\mathrm{H}_{2} \mathrm{~S}$, for a total of $57 \%$ ice. These ices could sublimate either during infall through the envelope, analogously to meteorites in Earth's atmosphere, or upon impact with the solid core. Helled et al. (2008) found that sublimation of volatiles during the collapse of a giant planet formed by disk instability (Boss 2005) is efficient: refractory silicate grains sediment to form a core, while ices remain in the planet atmosphere.

Tingle et al. (1991) tested the survival of volatiles experiencing hypervelocity impacts $\left(v \sim 1 \mathrm{~km} \mathrm{~s}^{-1}\right)$ by shocking samples of the Murchison meteorite with pressures up to $36 \mathrm{GPa}$. They found that $70 \%$ of organic and organosulfuric material, including $\mathrm{H}_{2} \mathrm{~S}$, sublimates upon experiencing an impact with $v>1.5 \mathrm{~km} \mathrm{~s}^{-1}$. The volatiles in Saturn-building planetesimals are not likely to survive a high-velocity impact and subsequent contact with the hot protoplanetary core, $T \geq 3000 \mathrm{~K}$, in solid form. If we assume all accreted ices undergo a phase transition from solid to gas, Saturn's core mass drops to $19 M_{\oplus}(\mathrm{S} 0)$ and $23 M_{\oplus}(\mathrm{S} 1)$, which are near the range determined by Saumon $\&$ Guillot (2004). However, the total heavy element/hydrogen mass ratio in the planet is still higher than observed: Saturn is
$50 \%-70 \%$ hydrogen by mass, whereas our model predicts a hydrogen mass fraction of only $42 \%$ (S0) and $31 \%$ (S1).

Another possible way to reduce Saturn's heavy element mass without slowing the planet's growth is to cut off solid accretion midway through planet formation. This approach simulates the effect of another embryo competing for planetesimals. Although our solid accretion rates are calculated for the monarchic growth paradigm, in which planetesimal dynamics are determined by proto-Saturn only, this scenario is an approximation: Kokubo \& Ida (1998) predict an oligarchic planet formation epoch with competing embryos spaced $\sim 10$ Hill radii apart that lasts for $\sim 1$ Myr.

Hubickyj et al. (2005) tested the effect of a core accretion cutoff on Jupiter's formation and found that as long as the core has mass $\geq 10 \quad M_{\oplus}$ before solid accretion ceases, the planet reaches hydrodynamic gas accretion even more quickly than when solid accretion continues unchecked: 0.78 Myr versus 2.22 Myr. Continuous, late-stage planetesimal accretion slows planet formation by depositing kinetic energy in the protoplanetary envelope and inhibiting gas contraction.

The competing embryo scenario holds promise for bringing Saturn's core/atmosphere mass ratio into agreement with observations while still retaining the quick formation time. However, it is a double-edged sword: oligarchs with overlapping zones of gravitational influence can increase the RMS planetesimal eccentricity, $\left\langle e^{2}\right\rangle^{1 / 2}$, far more efficiently than a single monarch core, decreasing the gravitational focusing ability of all embryos. Thommes et al. (2003) and Ida \& Makino (1993) find that significant planetesimal stirring can occur when embryos are between $10^{-5}$ and $10^{-2} M_{\oplus}$. Indeed, Fortier et al. (2007) replaced equation (2) with the analytical oligarchic growth rate of Ida \& Makino (1993) in in situ core accretion simulations of Jupiter and found formation times of 10-20 Myr.

Thommes et al. (2008) created a self-consistent planet formation model that included gas disk evolution, planet-disk interactions (including gap opening and gas accretion onto solid cores), and planet-planet interactions. Gas giants with Jupiterlike core/atmosphere mass ratios consistently emerged in disks with mass $M_{\text {disk }} \gtrsim 0.06 M_{\odot}$. Even a modest amount of migration, as occurred in the solar system, appears to compensate for the inefficiency of in situ oligarchic growth. The true Saturn formation scenario probably involved several $\sim 1 M_{\oplus}$ embryos spanning 9-12 AU, some outward motion of proto-Saturn, and possible competition with Neptune, forming near 12 AU (Tsiganis et al. 2005).

\subsection{Isolation Mass}

One new feature of both models, S0 and S1, is that Saturn does not need to accrete all the planetesimals in its feeding zone to reach its current mass. Figure 1 (right) shows the remaining solid surface density, not incorporated in Saturn's core, as a function of time. (The upturn near the end of the simulation results from the rapid expansion of the planet's feeding zone during hydrodynamic gas accretion and the assumption that planetesimals are always uniformly distributed, which is unphysical if the planet grows more quickly than planetesimal redistribution can occur.) In both models, there are still $2 \mathrm{~g}$ $\mathrm{cm}^{-2}$ of solids left in Saturn's feeding zone at $t=3.4 \mathrm{Myr}$. This means the core never reaches isolation mass. Following Lissauer (1993), we calculate

$$
M_{\text {iso }}=0.0021 \Sigma_{\text {solid }}^{3 / 2} a^{3}=58 M_{\oplus},
$$


whereas Saturn's core attains a mass of $44 M_{\oplus}$ in model S0 and $54 M_{\oplus}$ in model S1.

Both Hubickyj et al. (2005) and Pollack et al. (1996) found that giant planets pass through a lengthy plateau phase in which both solid and gas accretion rates are low and planet mass changes very little with time. This phase begins when the planet core nears isolation mass, and its duration is regulated by gas contraction efficiency. Examining Figure 1 (left), we see that Saturn never experiences this mass plateau: both solid and gas accretion rates increase with time.

With extra solid and gas mass remaining in the disk after 3.4 Myr, why would Saturn not continue to grow? We arbitrarily stop the simulation once Saturn reaches $95 M_{\oplus}$, but our disk could quickly form a more massive planet. Explaining Saturn's low mass in the context of the disk models in Paper I may require either another nearby embryo competing for both solids and gas-perhaps Neptune, forming near 12.5 AU according to the Nice model (Tsiganis et al. 2005)—or a mechanism for disk dissipation that begins after a few Myr. Photoevaporation (Alexander et al. 2006) is one possibility: the disk near $10 \mathrm{AU}$ begins to be disrupted by ionizing radiation at 2-3 Myr. We also require a mechanism to populate the Oort cloud with 40 $M_{\oplus}$ of comets (Weissman 1996). Saturn scattering the 4-14 $M_{\oplus}$ it does not accrete into the Oort cloud would be a good start, and Jupiter could contribute more material during the late stages of its formation and/or migration.

Is the concept of isolation mass relevant for any giant planet except Jupiter? From equation (3), we see that $M_{\text {iso }}$ increases with radius if $\alpha \leq 2$ in the power-law surface density profile $\Sigma \propto R^{-\alpha}$. Extrapolating from the simulation presented here and the near-flat solid surface density profile seen in Paper I, we propose that Saturn, Uranus, and Neptune do not reach isolation mass. Due to their low Keplerian orbital speed, planet cores in the outer solar nebula experience a lower planetesimal collision rate than proto-Jupiter. Instead of their formation timescale being governed by gas contraction efficiency, the solid accretion rate, mediated by $\Omega$ and $\Sigma$, is the critical factor. The hypothesis that the three outer planets never reach isolation mass is consistent with the planets' bulk composition: all three have solid/gas ratios far higher than Jupiter.

\section{CONCLUSIONS}

Based on the solid surface densities calculated in Paper I, we have created core accretion models of Saturn with a formation timescale of 3.37-3.48 Myr, which is compatible with observed protostellar disk lifetimes. Unlike previous studies of Jupiter (e.g., Hubickyj et al. 2005), we find that grain opacity in the protoplanetary envelope has virtually no effect on Saturn's formation timescale. The same solar nebula model that was the basis for simulations S0 and S1 is capable of forming Jupiter in $2.5 \mathrm{Myr}$, assuming reduced grain opacities.

Finally, the core accretion models presented here lack a plateau phase, in which the planet's mass remains nearly constant at approximately the isolation mass. We postulate that the low orbital speeds far from the Sun prevented Saturn, Uranus, and Neptune from accreting solids efficiently enough to reach isolation mass. These planets never experienced a gas-only accretion phase, as did Jupiter, and consequently have much higher core/atmosphere mass ratios.

We thank Geoff Bryden and Jonathan Fortney for helpful conversations and the anonymous referee for suggesting model S1. This research was supported by a scholarship award from the Achievement Rewards for College Scientists Foundation to S. D. R.; by NSF Career Grant AST-0449986 and NASA Planetary Geology and Geophysics Program Grant NNG04GK19G to G. L.; and by NSF Grant AST-0507424 and NASA Origins Grant NNX08AH82G to P. B. The work of K. W. and N. T. was carried out at the Jet Propulsion Laboratory, California Institute of Technology, under a contract with the National Aeronautics and Space Administration, with funding provided by the JPL Research and Technology Development Program.

\section{REFERENCES}

Alexander, R. D., Clarke, C. J., \& Pringle, J. E. 2006, MNRAS, 369, 229 Alibert, Y., Mousis, O., Mordasini, C., \& Benz, W. 2005, ApJ, 626, L57 Boss, A. 2005, ApJ, 622, 393

Dodson-Robinson, S. E., Willacy, K., Bodenheimer, P., Laughlin, G., Turner,

N. J., \& Beichman, C. 2008, Icarus, in press (arXiv:0806.3788) (Paper I) Flasar, F. M., et al. 2005, Science, 307, 1247

Fortier, A., Benvenuto, O. G., \& Brunini, A. 2007, A\&A, 473, 311

Haisch, K. E., Lada, E. A., \& Lada, C. J. 2001, ApJ, 553, L153

Helled, R., Podolak, M., \& Kovetz, A. 2008, Icarus, 195, 863

Henyey, L. G., Forbes, J. E., \& Gould, N. L. 1964, ApJ, 139, 306

Hubickyj, O., Bodenheimer, P., \& Lissauer, J. J. 2005, Icarus, 179, 415

Ida, S., \& Makino, J. 1993, Icarus, 106, 210

Kokubo, E., \& Ida, S. 1998, Icarus, 131, 171

Laughlin, G., Bodenheimer, P., \& Adams, F. C. 2004, ApJ, 612, L73

Lissauer, J. J. 1993, ARA\&A, 31, 129

Lunine, J. I., \& Stevenson, D. J. 1985, ApJS, 58, 493

Papaloizou, J. C. B., \& Nelson, R. P. 2005, A\&A, 433, 247
Papaloizou, J. C. B., \& Terquem, C. 1999, ApJ, 521, 823

Podolak, M. 2003, Icarus, 165, 428

Pollack, J. B., Hubickyj, O., Bodenheimer, P., Lissauer, J. J., Podolak, M., \& Greenzweig, Y. 1996, Icarus, 124, 62

Robinson, S. E., Laughlin, G., Bodenheimer, P., \& Fischer, D. A. 2006, ApJ, 643,484

Saumon, D., \& Guillot, T. 2004, ApJ, 609, 1170

Tanaka, H., Takeuchi, T., \& Ward, W. R. 2002, ApJ, 565, 1257

Thommes, E. W., Duncan, M. J., \& Levison, H. F. 2003, Icarus, 161, 431

Thommes, E. W., Matsumura, S., \& Rasio, F. A. 2008, Science, 321, 814

Tingle, T. N., Tyburczy, J. A., Ahrens, T. J., \& Becker, C. H. 1991, Origins of Life and Evolution of the Biosphere, 21, 385

Tsiganis, K., Gomes, R., Morbidelli, A., \& Levison, H. F. 2005, Nature, 435, 459

Weidenschilling, S. J. 1977, MNRAS, 180, 57

Weissman, P. R. 1996, Completing the Inventory of the Solar System, 107, 265 